\title{
Numerical Simulation of Fatigue Damage and Shape Instability Behavior of Steel 40Cr by the Damage-Coupled Crystal Plastic Model
}

\author{
G. C. Wu, Y. F. Li, X. D. Pan, and G. L. Wang
}

School of Mechatronics Engineering, Harbin Institute of Technology, Harbin, China

A representative volume element is developed based on the Voronoi tessellation to reveal the mechanism of shape instability behavior. In the model, a damage-coupled crystal plastic model is established to describe the shape instability behavior. The heterogeneity of materials is introduced into the model with the aim of simulating the microstructure of materials. The experimental and simulation results show that the fatigue damage in the elastic deformation stage with high cyclic stress level is the initial motivation of shape instability behavior. The cyclic softening and ratcheting properties of materials accelerate the plastic strain accumulated rate.

Keywords: shape instability failure mechanism, plastic deformation, finite element analysis, disposable mechanical elements.

Introduction. As the main failure mode for disposable mechanical elements that usually undertake critical cyclic loads, shape instability behavior is defined to describe the process from the total elastic deformation to the elastic-plastic deformation of materials [1] Figure 1 shows the stress-strain hysteresis cycle curves recorded in stress-controlled low-cycle fatigue tests. The shape instability failure is defined when the accumulated total plastic strain, $\varepsilon_{t}$, in the specimen reaches $0.2 \%$ in the fatigue process, $\varepsilon_{p}$ and $\varepsilon_{r}$ are cyclic plastic strain and ratcheting strain, respectively.

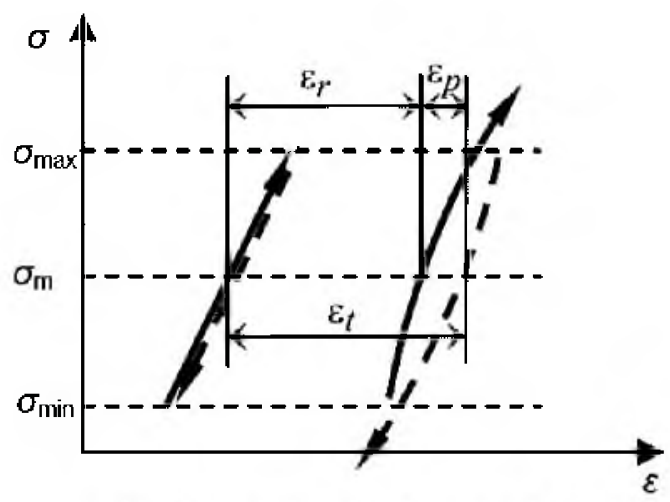

Fig. 1. Graph depicting the shape instability life.

The shape instability life data are vital for the reliability design of disposable mechanical elements. However, as shape instability failure that occurs during the transition from elastic deformation to plastic deformation concerns only the initial stage of plastic deformation, the shape instability life tests are fairly complex and inefficient. Therefore, in order to minimize the number of life tests and predict shape instability life, the mechanism of shape instability behavior should be firstly investigated to find out the main control factors. Shape instability behavior is similar to common ratcheting one. The latter phenomenon investigations are generally focused on the plastic evolution in the total fatigue process, and such phenomena are well understood [2-6]. Cyclic softening and 
fatigue damage can cause a similar behavior with shape instability [7-9]. However, as shape instability behavior is observed in the initial fatigue process, it is fairly difficult to search and observe inconspicuous microstructure evolution features in macrospecimens. Therefore, in order to reveal the mechanism of shape instability, the numerical method has to be used in investigations, although not as stand-alone application. In this study, the numerical method is independently used based on the similarity between the shape instability behavior and the ratcheting behavior. The proved results on the ratcheting behavior can be improved to establish a proper numerical model to simulate the shape instability behavior. Firstly, a three-dimensional cyclic crystal plastic model with inhomogeneity of material properties based on the cyclic plastic theory is developed to simulate the shape instability behavior. Then, the model parameters are modified by comparing the features of shape instability behavior described by the model and the experimental data. If the macrobehavior described by the numerical model is coincident with the one tested by the experiment data, it can be assumed that the numerical model represents the details of the shape instability process and the main factors controlling the shape instability behavior can be identified, which is instrumental for revealing the mechanism of shape instability behavior.

1. Materials and Specimens. In this study, steel $40 \mathrm{Cr}$ specimens were prepared for the parameter identification and shape instability experiments. The chemical composition of steel $40 \mathrm{Cr}$ is as follows (wt.\%): $0.45 \mathrm{C}, 0.5 \mathrm{Mn}, 0.37 \mathrm{Si}, 1.0 \mathrm{Cr}, 0.025 \mathrm{Ni}, 0.03 \mathrm{P}, 0.025 \mathrm{~S}$, and $0.03 \mathrm{Cu}$. The hardness of steel $40 \mathrm{Cr}$ bar was HRC $28-30$ by quenching and tempering treatment. Specimens with a gauge diameter of $12 \mathrm{~mm}$ and a gauge length of $25 \mathrm{~mm}$ were machined from steel bars with diameter of $22 \mathrm{~mm}$ from the same batch of hot-rolled steel $40 \mathrm{Cr}$. Turning and polishing processes were successively used to decrease and eliminate scratches at the gauge position and transition arc of the specimens. The roughness of the specimen gauges were measured on a surface profiler and the roughness value was no more than $0.4 \mu \mathrm{m}$. The cyclic stress-accumulated plastic strain curves were tested to determine the backstress and all other cyclic hardening parameters. Full tension- compression tests were conducted to obtain the fatigue life data and to determine the fatigue damage parameters.

2. Constitutive Model. In order to simulate the shape instability behavior, a proper cyclic crystal plasticity model should conclude both cyclic softening and fatigue damage parameters. It means that the constitutive model should be established based on a fatigue damage-coupled cyclic plasticity model. Therefore, a model based on the FrederickArmstrong model is used to describe the cyclic plastic deformation behavior of traditional metallic materials [10]. The evolution law of Ohno and Wang is combined with the framework of the Frederick-Armstrong model to adjust the ratcheting strain rate to improve the prediction accuracy of strain $[11,12]$. The elastic-plastic fatigue damage model provided by Lemaitre and Chaboche is involved to take the fatigue damage into consideration [13]. The main equations in the established model are presented as followed:

According to the small amounts of deformation theory, the total strain, $\varepsilon_{i j}$, is defined as

$$
\varepsilon_{i j}=\varepsilon_{i j}^{e}+\varepsilon_{i j}^{p},
$$

where $\varepsilon_{i j}^{e}$ is the elastic and $\varepsilon_{i j}^{p}$ is the plastic strains.

Value of $\varepsilon_{i j}^{e}$ is expressed as

$$
\varepsilon_{i j}^{e}=\frac{1+v}{E}\left(\frac{\sigma_{i j}}{1-D}\right)-\frac{v}{E}\left(\frac{\sigma_{k k} \delta_{i j}}{1-D}\right),
$$


where $v$ is the Poisson's ratio, $E$ is the elastic modulus, $\sigma_{i j}$ is the Cauchy stress tensor, $D$ is damage parameter, $\sigma_{k k}$ is the main diagonal component of $\sigma_{i j}$, and $\delta_{i j}$ is Kronecker delta.

The evolution law of plastic strain, $\widetilde{\varepsilon}_{i j}^{p}$, is defined as

$$
\widetilde{\varepsilon}_{i j}^{p}=\widetilde{\lambda} \frac{\partial F}{\partial \sigma_{i j}}
$$

where $\tilde{\lambda}$ is a non-negative scalar multiplier, $F$ is the Mises yield function for damage, which is as follows:

$$
F=\left(\frac{3}{2}\left(\frac{\sigma_{d e v}}{1-D}-\chi\right):\left(\frac{\sigma_{d e v}}{1-D}-\chi\right)\right)^{1 / 2}-Q,
$$

where $\sigma_{d e v}$ is the deviatoric stress tensor and $\chi$ is the backstress tensor. Value of $Q$ is the radius of the yield surface and defined as [14]:

$$
\widetilde{Q}=\tilde{p}\left(Q_{\infty}-Q\right) b_{1}
$$

where $Q_{\infty}$ and $b_{1}$ are material constants. Value of $\tilde{p}$ is defined as

$$
\tilde{p}=\left(\frac{2}{3}\left(\widetilde{\varepsilon}_{i j}^{p}: \widetilde{\varepsilon}_{i j}^{p}\right)\right)^{1 / 2}=\frac{\tilde{\lambda}}{1-D} .
$$

The backstress tensor $\chi$ in the Eq. (5) is expressed as

$$
\chi=\sum_{i=1}^{n} \chi^{(i)} \quad(i=1,2, \ldots, n)
$$

where $n$ is a backstress component, and $\chi^{(i)}$ is the respective total number of backstress components, defined as follows $[11,12]$ :

$$
\begin{gathered}
\dot{\chi}^{(i)}=(1-D) c^{(i)}\left[\frac{2}{3} r^{(i)} \dot{\varepsilon}^{p}-\left(\frac{\left\|\chi^{(i)}\right\|}{r^{(i)}}\right)^{m}\left\langle\dot{\varepsilon}^{p}: \frac{\chi^{(i)}}{\left\|\chi^{(i)}\right\|}\right) \chi^{(i)}\right], \\
\left\|\chi^{(i)}\right\|=\sqrt{\chi^{(i)}: \chi^{(i)}},
\end{gathered}
$$

where $c^{(i)}$ and $r^{(i)}$ are material constants and $m$ is an enhancement rate exponential.

Value of $D$ is calculated as follows [13]:

$$
\frac{\partial D}{\partial N}=\left[1-(1-D)^{1+\beta}\right]^{\alpha}\left(\frac{A_{I I}}{M_{0}\left(1-3 b_{2} \sigma_{H, \text { mean }}\right)(1-D)}\right)^{\beta},
$$

where $\alpha, \beta, M_{0}$, and $b_{2}$ are material constants and are determined based on the work of Bogard et al. [15], $\sigma_{H \text {, mean }}$ is the mean value of the hydrostatic stress, which is zero, and $A_{I I}$ is the parameter determined by the Sines yield criterion [16]. 
3. Model Development. The damaged-coupled cyclic crystal plastic model was programed by using the UMAT function of the software ABAQUS. The cyclic plastic strain and ratcheting strain in the initial fatigue process could be accurately predicted by using appreciate material property parameters. Voronoi tessellation is used to build a representative volume element. The features of material microstructures are approximately duplicated in the software ABAQUS.

Figure 2a, obtained via electron backscatter diffraction (EBSD) measurements, shows that the average grain size is $\sim 5 \mu \mathrm{m}$. As the steel $40 \mathrm{Cr}$ was hot rolled, the measured specimen was cut off from the small cylindrical bar along the radial direction. The grain size was then measured along the transverse direction. Because the grains in the material are generally equiaxed, each of the grain-size ratios, RD/ND and RD/TD, is assumed to be close to unity. The crystalline phase images confirm that the grains in the microstructure are equiaxed. According to the grain size, the grain density in the finite element model is approximately $8 \cdot 10^{6}$ polyhedrons $/ \mathrm{mm}^{3}$ in the simulation model. Obviously, the proper dimension for the model should be determined to reduce the analysis time. Therefore, the simulated zone is limited to a cube with an area of $50 \mu \mathrm{m}^{3}$ and 1000 polyhedrons. As shown in Fig. 2b, the polyhedrons in the cube are generated conforming to the rules of Voronoi tessellation. In Voronoi tessellation, the angles at the triple junctions may sometimes differ from $120^{\circ}$. In order to reduce the influence of the artificial stress, sharp angles at the junctions could be avoided by assigning seeds based on a uniform distribution. Only some of the junctions are triple junctions, and most of the angles at the junctions are not very sharp. In addition, the artificial concentration by angular differences at the triple junctions is insignificant as compared with that resulting from differences in the properties of the grains. This low concentration occurs because the total plastic strain is only $0.2 \%$.

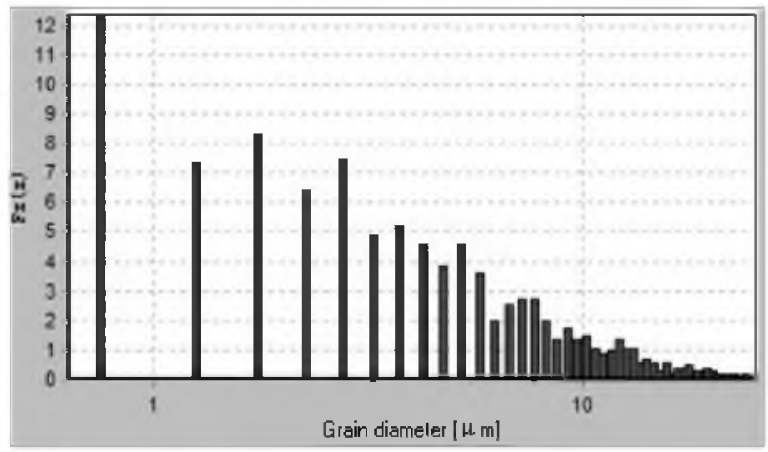

a

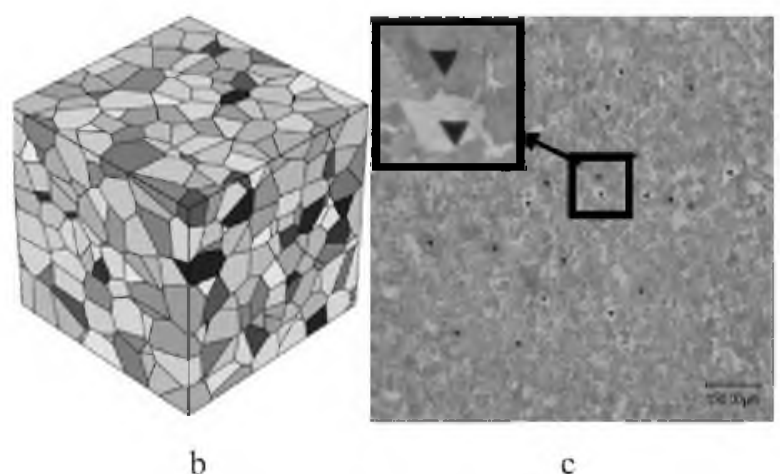

Fig. 2. The basic information used to simulate the micromechanical properties of grains: (a) grain size distribution in the region of interest from the EBSD measurement; (b) representative volume element of steel $40 \mathrm{Cr}$; (c) nanoindentation test conducted at 50 random points. 
Therefore, the error associated with the simulation is acceptable. By applying the periodic boundary condition on the cube, a representative volume element model is established. The grain mechanics properties are generated based on the stochastic distribution, which is determined according to nanoindentation test data. Figure $2 \mathrm{c}$ shows a part of 50 random positions on the specimen used to conduct the tests. The elastic module of the grains could thus be obtained directly. The yield strength of the grains could be experimentally determined from stress-strain curve of nanoindentation tests with relative ease based on the work of Liu and Chen [17]. These material data are then used to fit a Gaussian distribution. For the steel $40 \mathrm{Cr}$ in this study, the expected value and standard deviation of yield strength are 790 and $92 \mathrm{MPa}$, respectively. The effect of phases has been involved in the determination of distribution parameters and finally presented in the simulations of mechanical behaviors.

The cyclic crystal plasticity model is programmed and introduced into a representative volume element (RVE) model established in the software ABAQUS. As the stress range which is around the elastic limit of materials is fairly narrow, inhomogeneity of material properties is introduced into the RVE model to describe shape instability behavior. The RVE model with more realistic microstructure has the capacity to reflect the detail in the shape instability process. Figure 3 shows the method to incorporate the microstructural features and grain mechanics properties into the simulation model in ABAQUS. Figure $3 b$ shows the RVE model established in ABAQUS. Figure $3 b$ shows the procedure to assign random groups of material property data into the RVE model. A group of grain mechanics properties is randomly generated by using the Gaussian distribution function of ABAQUS. After that, the generated data are assigned to the grains in the RVE model. The established simulation model represents similar geometric features and mechanical properties as the real specimens on a micro level. By assigning the developed material constitutive model into the elements, the established RVE model is able to simulate the shape instability behavior.

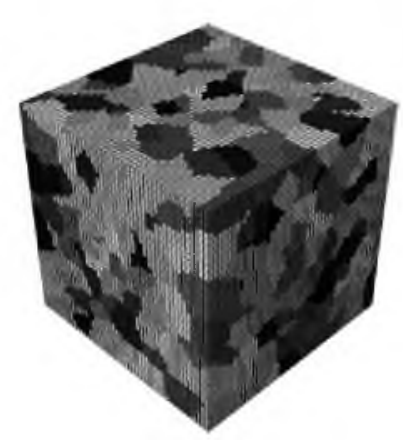

a

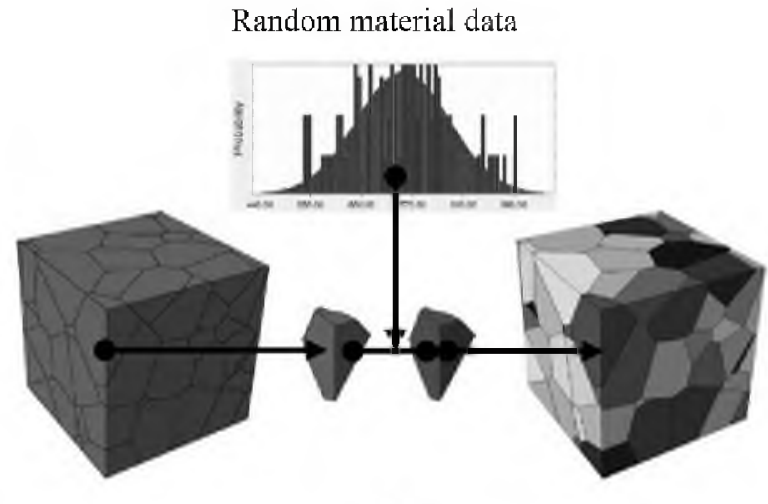

b

Fig. 3. Shape instability behavior simulation in ABAQUS: (a) representative volume element; (b) the method to introduce inhomogeneity of material properties into RVE.

4. Simulation and Experimental Results. Figure 4 shows the comparison results between experimental and simulated results. Generally, mean stress and stress amplitude are more frequently used in fatigue investigations. However, they are not intuitive parameters in the mechanical design. Maximum load and fatigue feature, corresponding to the maximum stress and stress ratio, are usually given as design indexes. Therefore, the experiment in this paper was conducted under stress ratios -1 and $-2 / 3$, and the constant maximum stress, which is equal to $95 \%$ of the yield strength. It is noted that the yield 
strength is usually set as an input parameter in normal finite element models. However, the random generation and assignment strategies of material properties in the grains of the model will all influence the macroperformance of the simulated yield strength, although the expected value of the material properties is the tested yield strength. Therefore, the simulated yield strength should be firstly simulated for the RVE model, but not directly specified as the test yield strength. The stress-strain curve with $0.2 \%$ total plastic strain is picked up from the whole fatigue process, both experimentally and via the simulations. Stress-strain hysteresis loops with similar features are picked out for both the experiments and the simulations by adjusting the material parameter $b_{1}$ from Eq. (6) and the damage parameter $D$. It means that these two parameters are the main factors influencing shape instability behavior.
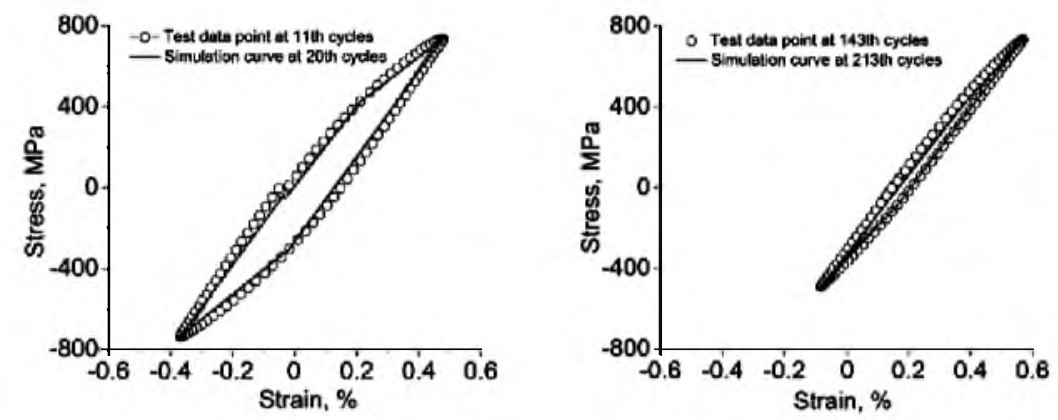

Fig. 4. Comparison results of steel $40 \mathrm{Cr}$ between experimental data and simulated results.

5. Discussion. Parameter $b_{1}$ represents the cyclic softening and ratcheting properties of materials. Damage parameter $D$ reflects the fatigue performance of materials. These two properties play different roles in the whole shape instability process. According to the plasticity deformation features, shape instability behavior could be divided into two stages including the plastic strain generation stage and the plastic strain increasing stage.

In the previous stage, according to the simulation model, the plastic strain is generated due to the fatigue damage of materials. In Eq. (6), the cyclic softening property of materials will cause plastic strain accumulation, only when the maximum stress is higher than the elastic limit, that is, the RVE model should be loaded in the plastic deformation zone (the constitutive models have been validated in many studies [18-20]). As the maximum stress in the simulation is the $95 \%$ of the elastic limit, it could be considered that the cyclic softening properties are not the trigger of shape instability behavior. In the micro level, the plastic strain occurs in the RVE model when the weakest grain exceeds its own elastic limit, although at this time the macro elastic limit of the RVE model is higher than the value of the weakest grain. It means that in the micro level the plastic strain in the RVE model is also irrelevant with the cyclic softening properties of materials. For the specimens in the experiment, flaws and defects are the weakest parts. The plastic strain will occur around these stress-concentration locations. Without fatigue damage, the accumulated plastic strain will tend to form stable hysteresis loops after a certain number of the symmetrical or unsymmetrical cyclic loading. Obviously, it is fairly difficult for materials arriving at $0.2 \%$ total plastic deformation only depending on the plastic strain from the stress-concentration. Therefore, it could be concluded that fatigue damage is primary in driving shape instability behavior at the initial stage.

In the plastic strain increasing stage, after the plastic strain is generated, the cyclic softening and ratcheting properties of materials will accelerate the accumulation rate of the plastic strain. The rapid increasing of plastic strain will lead to fatigue fracture much faster 
than expected. In this stage, neglecting cyclic softening and ratcheting properties will overestimate the fracture life and lead to fatal risks.

As the limitation of experiment observation methods, the conclusion is not obtained based on the experiment result but from the numerical simulation. However, the conclusion is credible, because the numerical model is established based on the previous proved theories according to the similarity between the shape instability behavior and the ratcheting behavior. Since the mechanics behavior in macrolevel could be described by using the numerical model, it is reasonable to assume that the detail in the numerical model is similar with the material microstructures in the microlevel, therefore, the numerical simulation results are reliable.

Conclusions. The investigation shows that the fatigue damage in the elastic deformation stage with high stress level is the initial motivation of shape instability behavior. The cyclic softening and ratcheting properties of materials accelerate the plastic strain accumulated rate. Shape instability behavior is not the essential property of cyclic softening materials. It explains that some cyclic softening materials, such as Ti-6Al-4V, do not show shape instability behavior. If cyclic softening materials have excellent fatigue performance in the elastic deformation stage, shape instability behavior could be ignored. It means that it is not necessary to test the shape instability life of these cyclic softening materials.

Acknowledgments. This study is supported by the National Natural Science Foundation of China (51405101), the research and innovation fund of Harbin Institute of Technology (Grant Number HIT.NSRIF.2015 053), the China Postdoctoral Science Foundation (Grant Numbers 2014M561340 and 2016T90277) and Heilongjiang Postdoctoral Fund (Grant Number LBH-Z14100).

1. Y. F. Li, X. D. Pan, and G. L. Wang, "Low cycle fatigue and ratcheting properties of steel 40Cr under stress controlled tests," Int. J. Fatigue, 55, 74-80 (2013).

2. S. J. Park., K. S. Kim, and H. S. Kim, "Ratcheting behaviour and mean stress considerations in uniaxial low-cycle fatigue of Inconel 718 at $649^{\circ} \mathrm{C}$," Fatigue Fract. Eng. Mater. Struct., 30, 1076-1083 (2007).

3. C. B. Lim, K. S. Kim, and J. B. Seong, "Ratcheting and fatigue behavior of a copper alloy under uniaxial cyclic loading with mean stress," Int. J. Fatigue, 31, 501-507 (2009).

4. G. Z. Kang, Y. J. Liu, J. Ding, and Q. Gao, "Uniaxial ratcheting and fatigue failure of tempered 42CrMo steel: Damage evolution and damage-coupled viscoplastic constitutive model," Int. J. Plasticity, 25, 838-860 (2009).

5. H. Lim, J. D. Carroll, C. C. Battaile, et al., "Grain-scale experimental validation of crystal plasticity finite element simulations of tantalum oligocrystals," Int. J. Plasticity, 60, 1-18 (2014).

6. D. Yu, K. An, Y. Chen, and X. Chen, "Revealing the cyclic hardening mechanism of an austenitic stainless steel by real-time in situ neutron diffraction," Scripta Mater., 89, 45-48 (2014).

7. S. Keshavarz and S. Ghosh, "Multi-scale crystal plasticity finite element model approach to modeling nickel-based superalloys," Acta Mater., 61, 6549-6591 (2013).

8. M. Naderi, S. H. Hoseini, and M. M. Khonsari, "Probabilistic simulation of fatigue damage and life scatter of metallic components," Int. J. Plasticity, 43, 101-115 (2013).

9. S. Ghosh and M. Anahid, "Homogenized constitutive and fatigue nucleation models from crystal plasticity FE simulations of Ti alloys, Part 1: Macroscopic anisotropic yield function," Int. J. Plasticity, 47, 182-201 (2013). 
10. C. O. Frederick and P. J. Armstrong, A Mathematical Representation of the Multiaxial Bauschinger Effect, Report RD/B/N 731, Central Electricity Generating Board (1966).

11. N. Ohno and J.-D. Wang, "Kinematic hardening rules with critical state of dynamic recovery, part I: formulation and basic features for ratchetting behavior," Int. J. Plasticity, 9, 375-389 (1993).

12. N. Ohno and J.-D. Wang, "Kinematic hardening rules with critical state of dynamic recovery, part II: Application to experiments of ratchetting behavior," Int. J. Plasticity, 9, 391-403 (1993).

13. J. Lemaitre and J. L. Chaboche, Mechanics of Solid Materials, Cambridge University Press, Cambridge (1990).

14. G. Z. Kang, Q. Gao, L. X. Cai, and Y. F. Sun, "Experimental study on uniaxial and nonproportionally multiaxial ratcheting of SS304 stainless steel at room and high temperatures," Nucl. Eng. Des., 216, 13-26 (2002).

15. F. Bogard, P. Lestriez, and Y. Q. Guo, "Damage and rupture simulation of mechanical parts under cyclic loading," J. Eng. Mater. Technol., 132, 0210031-0210038 (2010).

16. G. Sines and G. Ohgi, "Fatigue criteria under combined stressee or straine," J Eng. Mater. Technol., 103, 82-90 (1981).

17. Y. Liu and D. Chen, "Measurement of material mechanical properties using nanoindentation and finite element simulation," J. Wuhan Univ. Technol., 27, 690-693 (2003).

18. N. Ohno, "Constitutive modeling of cyclic plasticity with emphasis on ratcheting," Int. J. Mech. Sci., 40, 251-261 (1998).

19. N. Ohno, M. Abdel-Karim, M. Kobayashi, and T. Igari, "Ratchetting characteristics of 316FR steel at high temperature, part I: strain-controlled ratchetting experiments and simulations," Int. J Plasticity, 14, 355-372 (1998).

20. N. Ohno and M. Abdel-Karim, "Uniaxial ratchetting of $316 \mathrm{FR}$ steel at room temperature - Part II: Constitutive modeling and simulation," J Eng. Mater. Technol., 122, 35-41 (2000). 